\title{
INTESTINAL PARASITOSIS AND PEDIATRIC HEPATIC DISEASE: COPROSCOPY AND IMMNO MOLECULAR ASSAYS
}

\author{
By \\ MARWA A. GHIETH ${ }^{1 *}$, AYMAN A. EL-BADRY ${ }^{2}$, ENAS Y. ABU-SAREA ${ }^{1}$ \\ And MAGD A. KOTB ${ }^{3}$
}

Department of Medical Parasitology ${ }^{1}$, Faculty of Medicine, Beni-Suef University, Beni-Suef, Egypt, Department of Microbiology ${ }^{2}$, College of Medicine, University of Dammam, Dammam, Saudi Arabia, Department of Pediatrics ${ }^{3}$, Faculty of Medicine, Cairo University, Giza, Egypt ( ${ }^{*}$ Correspondence: marwaghieth@yahoo.com)

\begin{abstract}
Parasitic infections in children with liver diseases could be a serious health problem. This study documented a true prevalence of intestinal parasitosis in children with pediatric liver diseases and estimate risk factors for parasitic infection. Faecal samples were collected from 95 children suffering liver diseases. Samples were examined microscopically for parasitic stages and coproscopy of acid fast (AF) stained fecal smear for Cryptosporidium oocysts, by ELISA for E. histolytica and Giardia and by PCR genotyping for E. histolytica and Cryptosporidium spp. copro-DNA. G. intestinalis was the prevailing protozoa $(14.7 \%)$ followed by Hymenolepis nana $(8.4 \%)$ and $C$. hominis $(4.2 \%)$. The pathogenic $E$. histolytica was not detected by genetic differentiation or ELISA. Only age group ( $P$ value .004$)$ was a risk factor for intestinal parasitosis among these children. Giardiasis, hymenolepiasis nana and cryptosporidiosis are the most prevailing parasites in children with liver disease that physicians must consider. Accurate diagnosis using molecular technique is a must with E.histolytica and helpful with Cryptosporidium genotyping for epidemiological purposes.
\end{abstract}

Key words: Intestinal parasitosis, Liver diseases, Diarrhea, nPCR, RFLP, Genotyping

\section{Introduction}

Liver diseases could impair cell mediated immunity making hepatic patients more susceptible to infections (Zhang et al, 2008; Youssef et al, 2008). Opportunistic parasites in hepatic patients may lead to severe diarrhea, dehydration and electrolyte imbalance (Tuli et al. 2010). Intestinal parasitosis with E.histolytica, Cryptosporidium spp. and $G$. intestinalis caused severe illness in immunocompromised patients (Nazeer et al. 2013). E. histolytica is manifested by diarrhea, dysentery or extraintestinal complications such as amoebic liver abscess which are fatal, hence accurate diagnosis is vital (Petri et al. 2000; Ohnishi et al, 2004; Zebardast et al, 2016). The Tech-Lab E. histolytica coproantigen detection kit was properly diagnosed E. histolytica (WHO, 1997). For true estimation of pathogenic amoebiasis molecular techniques are required (Parija et al, 2010; Lu et al, 2016).

Cryptosporidiosis is a known cause of diarrhea for both patients with impaired immunity and healthy. Dissemination to other organs is a risk in immunocompromised pa- tients (Snelling et al, 2007; Stark et al, 2011; Goñi et al, 2012). Cryptosporidium was detected by Shrestha et al. (1993) and ElShazly et al. (2015) used microscopic examination of stool in patients with liver diseases (20\% \& 10\%, respectively) and Mousa et al. (2014) reported to be an aggravating factor for hepatic encephalopathy.

G. intestinalis is a major cause of parasitic diarrhea in children. The course of giardiasis may be prolonged to chronic diarrhea, weight loss and malabsorption in patients with impaired immunity (Muhsen and Levine, 2012; Helmy et al, 2014).

The present study focused on a group of children suffering liver diseases, to explore the parasitosis prevalence among them using immune-assay and molecular techniques, and to monitor risk factors for parasitosis.

\section{Material and Methods}

Ethical considerations: All patients included were informed verbally about the purpose of the study and their parents' consent was taken before collection of stool samples.

Study population: The cross-sectional study was carried out on 95 children aged from 
1-15 years old attending the Pediatric Hepatology Unit, Cairo University Pediatrics Hospitals (CUPH) and proved by clinical, radiological and/or laboratory tests to have liver diseases. None of them were having liver cell failure. All patient related demographic and clinical data were collected using designed questionnaires.

Collection and processing of samples: From 1 to 3 stool samples were collected from all children and divided into three parts, one for examination by direct wet mount and formalin-ethyl acetate concentration after adding saline and Lugol's iodine to detect the intestinal protozoans (oo)cysts, helminthes ova and other parasitic stages using x10 \& x40 objectives. Modified Ziehl Neelsen stain was used to detect Cryptosporidium oocyst. The other two parts were freshly frozen at $-20^{\circ} \mathrm{C}$ for further assays.

Copro-immunoassay: Parts of the frozen samples were subjected to coproantigen detection of E.histolytica using Wampole TM E.histolytica II, $2^{\text {nd }}$ generation monoclonal ELISA kit (Tech-Lab, Blacksburg, Virginia, USA, No.T5017) and G. intestinalis using RIDASCREEN (R-Biopharm GmbH, Darmstadt, Germany) kit according to the manufacturer`s instructions.

Copro-PCR assay: It was used for detection and genotyping of Cryptosporidium targeting Cryptosporidium oocyst wall protein (COWP) gene using nPCR Restriction fragment length polymorphism (RFLP) and genetic differentiation of Entamoeba complex using 16S-like gene. All samples were subjected to genomic DNA extraction using Favor Prep DNA isolation Kit (Favorgen Biotech, Taiwan, Cat. No. FASTI001-1) according to the manufacturer's instructions with some modifications, using alternation of liquid nitrogen thermal shock followed by $95^{\circ} \mathrm{C}$ water bath for 10 cycles.

PCR amplification: For detection of Cryptosporidium two sets of primers were used. BCOWPF (5'-ACCGCTTCTCAACAACCA TCTTGTCCTC-3'), BCOWPR (5'-CGCAC CTGTTCCCACTCAATGTAAACCC-3') at 796bp fragment (Pedraza-Díaz et al, 2000). Nested primers, cry-15 (5'-GTAGATAATG GAAGAGATTGTG-3'), cry-9 (5'-GGACTGAAATACAGGCATTATCTTG-3') at 553 bp fragment were used. The reaction conditions and mixture were done (Spano et al, 1997). PCR amplified products were visualized by using ethidium bromide stain, agarose gel electrophoresis $(1.5 \%)$ \& UV Transilluminator. Positive PCR products were digested by RsaI (Fer-mentas UAB, V. Graiciuno 8, LT-02241 Vilnius, Lithuania), digested N-COWP frag-ments were resolved by electrophoresis in typing-grade agarose gels $(3.2 \%)$ stained with ethidium bromide, the fragments were visualized by UV light to determine Cryptosporidium genotype.

For genetic differentiation of $E$. histolyti$c a$, E. dispar and E. moshkovskii a multiplex PCR (mPCR) targeting 16S-like gene was used, for primary reaction EF 5'-TAA GAT GCA GAG CGA AA-3' \& ER 5'-GTA CAA AGG GCA GGG ACG TA-3' were used at $\sim 800 \mathrm{bp}$ amplification. For secondary reaction the following sets of primers were used EHF 5'-AAG CAT TGT TTC TAG ATC TGA G-3' and EHR 5'-AAG AGG TCT AAC CGA AAT TAG-3' at a fragment of $\sim 439 \mathrm{bp}$ for E. histolytica, EDF 5'TCT AAT TTC GAT TAG AAC TCT-3' and EDR 5'-TCC CTA CCTATT AGA CAT AGC-3'at a bp of $\sim 174$ for $E$. dispar and EMF 5'-GAA ACC AAG AGT TTC ACA AC-3' and EMR 5'-CAA TAT AAG GCT TGG ATG-3' at a bp of $\sim 553$ for $E$. moshkovskii with reaction components and cycling conditions (Ngui et al, 2012). The amplified products were visualized with agarose gel electropho-resis (1.5\%) stained with ethidium bromide.

Statistical analysis: Data were tabulated, coded and analyzed with the SPSS version 20 (statistical package for social science). Qualitative variables were analyzed by percentage and frequency, while quantitative variables were described by mean \pm SD. Risk factors of intestinal parasitosis were the dependent variable among the studied popu- 
lation, odds ratio (OR), 95\% confidence interval $(\mathrm{CI}) \& P$ value $<0.05$ was significant.

\section{Results}

The study included 95 children, 57 (60\%) male and $38(40 \%)$ female with a mean age of 6.1 3 3.4. Among them, $40(42.1 \%)$ patie- nts were harboring one parasite at least; while the remaining $55(57.9 \%)$ patients were free. Results of parasitic prevalence, diagnostic yielding, demographic, clinical data and hepatic etiology were given (Tabs. $1,2,3,4 \& 5)$.

Table 1: Coproscopic prevalence of parasitic infections among studied population.

\begin{tabular}{|l|l|l|}
\hline Parasitic infection & Total $(\mathrm{n}=95)$ and \% \\
\hline Protozoa & Giardia cysts/trophozoites & $13(13.6)$ \\
\cline { 2 - 3 } & E. complex cysts/trophozoites & $4(4.2)$ \\
\cline { 2 - 3 } & Entamoeba coli cysts & $3(3.1)$ \\
\cline { 2 - 3 } & Blastocyst hominis cysts & $4(4.2)$ \\
\cline { 2 - 3 } & Entamoeba coli / Blastocyst hominis cysts & $3(3.1)$ \\
\cline { 2 - 3 } & Cryptosporidium oocysts (MZN stain) & $1(1.1)$ \\
\cline { 2 - 3 } & Iodamoeba butchii cysts & $1(1.1)$ \\
\hline Helminthes & Hymenolips nana eggs & $8(8.4)$ \\
\cline { 2 - 3 } & Taenia eggs & $2(2.1)$ \\
\cline { 2 - 3 } & Entrobius vermicularis & $1(1.1)$ \\
\hline
\end{tabular}

Table 2: Prevalence of G.intestinalis and molecular prevalence of E. complex \&Cryptosporidium spp. among population.

\begin{tabular}{|c|c|c|c|c|c|}
\hline $\begin{array}{c}\text { Positive } \\
\text { cases }\end{array}$ & Giardia by microscopy and ELISA n (\%) & Cryptosporidium by nPCR n $(\%)$ & \multicolumn{3}{|c|}{$E$. complex by mPCR } \\
\cline { 3 - 6 } $\begin{array}{c}\text { Total } \\
(\mathrm{n}=95)\end{array}$ & $14(14.7)$ & $4(4.2)$ & $E . h^{*}$ & $E . d^{*}$ & $E . m^{*}$ \\
\hline
\end{tabular}

E.h: E.histolytica, E.d: E.dispar and E.m: E.moshkovskii

Table 3: Age groups distributions among studied population

\begin{tabular}{|l|l|l|l|l|}
\hline \multirow{2}{*}{ Age groups } & Intestinal parasitosis & \multirow{2}{*}{ Total $(\mathrm{n}=95)$} & \multirow{3}{*}{$P$ value } \\
\cline { 2 - 4 } & Yes $(\mathrm{n}=40)$ & No $(\mathrm{n}=55)$ & & \multirow{2}{*}{$.004 *$} \\
\cline { 2 - 4 } & $\mathrm{n}(\%)$ & $\mathrm{n}(\%)$ & $\mathrm{n}(\%)$ & \\
\hline Infant (up to 2 years) & $2(5)$ & $9(16.3)$ & $11(11.6)$ \\
\hline Early childhood (2-6years) & $15(37,5)$ & $29(52.7)$ & $44(46.3)$ \\
\hline Late childhood (6-12 years) & $19(47.5)$ & $15(27.3)$ & $34(35.7)$ \\
\hline Young teens (12-15 years) & $4(10)$ & $2(3.6)$ & $6(6.3)$ & \\
\hline
\end{tabular}

Table 4: Demographic and clinical features of studied population.

\begin{tabular}{|c|c|c|c|c|c|c|c|c|}
\hline \multirow{3}{*}{\multicolumn{2}{|c|}{ Variable }} & \multicolumn{2}{|c|}{ Intestinal parasitosis } & \multirow{2}{*}{ Total $(n=95)$} & \multirow{3}{*}{$\begin{array}{l}P \\
\text { value }\end{array}$} & \multirow[t]{3}{*}{ *OR } & \multirow{2}{*}{\multicolumn{2}{|c|}{$* * 95 \% \mathrm{CI}$}} \\
\hline & & Yes $(n=40)$ & No $(n=55)$ & & & & & \\
\hline & & \multirow{2}{*}{$\begin{array}{l}23(57.5) \\
17(42.5)\end{array}$} & \multirow{2}{*}{$\begin{array}{l}34(61.8) \\
21(38.2)\end{array}$} & \multirow{2}{*}{$\begin{array}{l}57(60) \\
38(40)\end{array}$} & & & lower & upper \\
\hline Giardia & $\begin{array}{l}\text { Male } \\
\text { Female }\end{array}$ & & & & .41 & .83 & .364 & 1.91 \\
\hline \multirow{5}{*}{$\begin{array}{l}\text { Gastro- } \\
\text { intestinal } \\
\text { symp- } \\
\text { toms }\end{array}$} & Diarrhea & $24(60)$ & $35(63.6)$ & $59(62.1)$ & .83 & .85 & .371 & 1.981 \\
\hline & Abdominal pain & $12(30)$ & $12(21.8)$ & $24(25.3)$ & .47 & 1.53 & .605 & 3.896 \\
\hline & Fatigue & $16(26.6)$ & $10(28.5)$ & $26(27.3)$ & .25 & .64 & .254 & 1.656 \\
\hline & Flatulence & $8(20)$ & $5(9.1)$ & $13(13.6)$ & .14 & 2.50 & .751 & 8.31 \\
\hline & Dysentery & $6(10)$ & $3(8.5)$ & $9(9.4)$ & .45 & 1.92 & .406 & 9.129 \\
\hline
\end{tabular}

Table 5: Hepatic etiology among studied population.

\begin{tabular}{|l|c|c|c|}
\hline \multirow{2}{*}{ Hepatic diseases } & Intestinal parasitosis & \multirow{2}{*}{$\begin{array}{l}\text { Total } \\
(\mathrm{n}=95)\end{array}$} & P value \\
\cline { 2 - 3 } \cline { 2 - 3 } & Yes $(\mathrm{n}=40) \%$ & No $(\mathrm{n}=55) \%$ & $46(48.4)$ \\
\hline Metabolic disease & $16(40)$ & $30(54.5)$ & $11(11.5)$ \\
\hline Hepatitis C & $5(12.5)$ & $6(10.9)$ & $7(7.3)$ \\
\hline Hepatitis B & $4(10)$ & $3(5.4)$ & $3(3.1)$ \\
\hline Hepatitis C\&B & $2(5)$ & $1(1.8)$ & $9(9.4)$ \\
\hline Hepatitis A & $3(7.5)$ & $6(10.9)$ & $5(5.2)$ \\
\hline Autoimmune disease & $3(7.5)$ & $2(3.6)$ & $4(4.2)$ \\
\hline Wilson disease & $2(5)$ & $2(3.6)$ & $4(4.2)$ \\
\hline Congenital hepatic fibrosis & $2(5)$ & $2(3.6)$ & $4(4.2)$ \\
\hline Fasciola infection & $2(5)$ & $2(3.6)$ & $2(2.1)$ \\
\hline Cystic fibrosis & $1(2.5)$ & $1(1.8)$ & \\
\hline
\end{tabular}

\section{Discussion}

In the present study, G. intestinals (14.7

$\%)$ was the most prevalent protozoa among children suffering hepatic diseases followed by Hymenolepis nana ( $8.4 \%)$. Reduced immunity caused by the liver affection could 
play a role in transmission of parasitic infections in addition to the socioeconomic and environmental factors, as a number of intestinal parasites and commensals beside $G$. intestinalis, Hymenolepis nana and C. hominis were detected. Hegab et al. (2003) reported that $G$. intestinals $(45 \%)$ was the commonest parasite in children with chronic liver disease. Also, Nazeer et al. (2013) using real-time PCR found that giardiasis (37.1\%) was the commonest one followed by Cryptosporidium spp (3\%).

In the present study, cryptosporidiosis was the second protozoa (4.2\%), all positive cases of $C$. hominis, three of whom were complaining of diarrhea. Predominance of the anthroponotic strain $C$. hominis supported man to man transmission by water contamination. Using nPCR targeting COWP gene detected $75 \%$ of cases which were negative by microscopy. Fathy et al. (2014) and El-Badry et al. (2015) reported the precious use of molecular techniques in cryptosporidiosis diagnosis. Abd El Kader et al. (2012) and El-Badry et al. (2015) reported the predominance of C. hominis $(80 \% \& 95.8 \%$, respectively) among Egyptian diarrheic patients. But, Eraky et al. (2014) found the predominance of $C$. parvum $(82 \%)$ and Mousa et al. (2014) found a higher rate of $C$. parvum than $C$. hominis in chronic liver disease patients. Differences might point to the sources of infection and features of water supply contaminations in different communities which vary from one area to another.

The prevalence of Entamoeba complex by microscopy was $4.2 \%$, all suffered from diarrhea without dysentery. However, pathogenic E. histolytica was not detected by 2 nd generation monoclonal ELISA kit (Tech Lab) or by molecular differentiation using 16S-like gene. Also, neither E. dispar nor $E$. moshkovskii were detected by PCR, suggested that positive microscopy cases were related to other species like E. polecki, E. hartmanni or E. bangladeshi. Nazeer et al. (2013) reported a microscopic prevalence of $10.8 \%$ for E. histolytica/dispar among 396 diarrheic patients, without E. histolytica by real-time PCR.

But, Younes et al. (1996), Hegab et al. (2003) and El-Shazly et al. (2015) reported high microscopic prevalence of E. histolytica/E.dispar $(21.5 \%, 37.5 \%$ \& $16 \%$, respectively) among patients with chronic liver diseases and recurrent diarrhea. This discrepancy between studies could be explained by the differences in the diagnostic methods used. Microscopy was a non-specific method and led to false positive results and could not differentiate the pathogenic amoebiasis from other morphologically identical nonpathogenic species (Parija et al, 2010; Zebardast et al, 2016). Thus microscopy results carried the risk of exposure of healthy children to unnecessary treatment (Haque et al. 2006).

Healthy intestinal epithelium and normal tight junction represent natural barriers for gut immunity (Vajro et al, 2013). Commensals are major component of gut microbiota which in role is vital for keeping gut mucosal barriers integrity (Mohajeri et al, 2018). The present patients were free from liver cell failure reflecting a controllable state of liver, a question arises are the nonpathogenic amoeba spp and other commensals detected among our study population helpful to patients with liver disease? Or even they might contribute for better microbiota and keeping gut integrity, non-specific treatment will be even harmful to these patients.

The commonest hepatic etiology among the studied population was metabolic liver diseases $(48.4 \%)$, followed by hepatitis C, A \& B $(11.5 \%, 9.4 \%$ \& $7.3 \%$, respectively). However patients with cystic fibrosis $(2.1 \%)$ were the least represented. Not all patients were complaining of chronic liver diseases, acute hepatitis A represented $9.4 \%$ of the patients. All patients weren't complaining of liver cell failure. Liver etiology wasn't significantly a risk factors for parasitosis $(P=$ .398). Among studied variables to determine the predictive factors for intestinal parasitosis among children with hepatic disease, on- 
ly age group $(P=.004)$ was a significant risk factor. Intestinal parasitosis among different age groups of hepatic children reached its peak during late childhood (6-12 years old = $47.5 \%$ ) which represented the age of grade scholars where the children use toilets in school and public regions and didn't care about their hygiene and neglect washing hands before meals in addition, they used to play and eat outdoors being more exposed to parasitic infections. Followed by the age of early childhood (2-6years $=37.5 \%$ ), the preschool age, as children may attend day care centers and nursery schools being more exposed to infectious parasites through eating or drinking. The least exposed group was the infantile age (up to years old $=5 \%$ ) as they depend on their mother on feeding being less exposed to infected food. Young teens (up to 15 years old $=10 \%$ ) were personal hygiene of children improved by this age.

\section{Conclusion}

$G$. intestinals, $H$. nana and $C$. hominis must be suspected among children with liver diseases. Once liver is free from cell failure, hepatic etiology isn't a risk factor for intestinal parasitosis. Microscopic positive E. complex must be confirmed molecularly for pathogenic E. histolytica to avoid unnecessary treatment or disturbance of gut microbiota. Genotyping of Cryptosporidium proved essential for targeting the infection source.

\section{Acknowledgment}

The authors are grateful to the Scientific Research Developing Unit, Beni-Suef University for granting and funding the study given to Dr. Ghieth. Also, to the Diagnostic \& Research Unit of Parasitic Diseases (DRUP) and Lab of Molecular Medical Parasitology (LMMP) Medical Parasitology Department, Faculty of Medicine, Cairo University, for processing of samples in addition to the Pediatric Hepatology Unit, Faculty of Medicine, Cairo University.

Compliance with Ethical Standards: Protocol was approved by Beni-Suef University Research Ethics Committee. Sample collections and ethics were in agreement with the
Helsinki declaration; 1964.

Informed consent: All patients were informed verbally about the study purpose and their parents' consent was taken before collection of stool samples.

\section{Conflict of interest: None.}

\section{References}

Abd El Kader, NM, Blanco, MA, Ali-Tammam, M, Abd El Ghaffar, AR, Osman, A, et al, 2012: Detection of Cryptosporidium parvum and Cryptosporidium hominis in human patients in Cairo, Egypt. Parasitol. Res. 110, 1:161-6.

El-Badry, AA, Al-Antably, AS, Hassan, MA, Hanafy, NA, Abu-Sarea, EY, 2015: Molecular seasonal, age and gender distributions of cryptosporidium in diarrhoeic Egyptians: distinct endemicity. Euro J. Clin. Microbiol. Infect. Dis. 34, 12:2447-53.

El-Shazly, LB, El-Faramawy, AA, El-Sayed, NM, Ismail, KA, Fouad, SM, 2015: Intestinal parasitic infection among Egyptian children with chronic liver diseases. J. Parasit. Dis. 39, 1:7-12

Eraky, MA, El-Hamshary, AM, Hamadto, H H, Abdallah, KF, Abdel-Hafed, WM, et al, 2014: Predominance of Cryptosporidium parvum genotype among diarrheic children from Egypt as an indicator for zoonotic transmission. Acta Parasitol. 60, 1:26-34,

Fathy, MM, Abdelrazek, NM, Hassan, FA, El-Badry, AA, 2014: Molecular copro prevalence of Cryptosporidium in Egyptian children and evaluation of three diagnostic methods. Indian Pediat. 51, 9:727-9.

Goñi, P, Martín, B, Villacampa, M, García, A, Seral, C, Castil et al, 2012: Evaluation of an immunochromatographic dip strip test for simultaneous detection of Cryptosporidium spp, Giardia duodenalis, and Entamoeba histolytica antigens in human fecal samples. Euro J. Clin. Microbiol. Infect. Dis. 31, 8:2077-82.

Haque, R, Mondal, D, Duggal, P, Kabir, M, Roy, S, Farr, BM, et al, 2006: Entamoeba his tolytica infection in children and protection from subsequent amebiasis. Infect. Immun. 74:904-9.

Hegab, MH, Zamzam, SM, Khater, NM, Tawfeek, DM, AbdelRahman, HM, 2003: Opportunistic intestinal parasites among children with chronic liver disease. J. Egypt. Soc. Parasitol. 33:969-77.

Helmy, YA, Klotz, C, Wilking, H, Krucken, J, Nockler, K, et al, 2014: Epidemiology of Giardia duodenalis infection in ruminant livestock 
and children in the Ismailia province of Egypt: insights by genetic characterization. Parasit. Vectors 7:321-8.

Lu Y, Chen, J, Zhang, Y, et al, 2016: Identification of Entamoeba histolytica by FTA-nested PCR. Chin. J. Zoono. 32, 2:128-32.

Mohajeri, MH, Brummer, RJM, Rastall, RA, et al, 2018: The role of the microbiome for human health: from basic science to clinical applications. Eur. J. Nutr. 57, 1:1-4.

Mousa, N, Abdel-Razik, A, El-Nahas, H, ElShazly, A, Abdelaziz, M, et al, 2014: Cryptosporidiosis in patients with diarrhea and chronic liver diseases. J. Infect. Develop. Count. 8, 12: 1584-90.

Muhsen, K, Levine, MM, 2012: A systematic review and meta-analysis of the association between Giardia lamblia and endemic pediatric diarrhea in developing countries. Clin. Infect. Dis. 55, 4:S271-93

Nazeer, JT, Khalifa, KE, Von Thien, H, ElSibaei, MM, Abdel-Hamid, MY, et al, 2013: Use of multiplex real-time PCR for detection of common diarrhea causing protozoan parasites in Egypt. Parasitol. Res. 112, 2:595-601.

Ngui, R, Angal, L, Fakhrurrazi, SA, Lian, YA, Ling, LY, et al, 2012: Differentiating Entamoeba histolytica, Entamoeba dispar and Entamoeba moshkovskii using nested polymerase chain reaction (PCR) in rural communities in Malaysia. Parasit. Vectors 5:187-91.

Ohnishi, KY, Kato, A, Imamura, M, Fukayama, T, Tsunoda, Y, et al, 2004: Present characteristics of symptomatic Entamoeba histolytica infection in the big cities of Japan. Epidemiol. Infect. 132:57-60.

Parija, SC, Garg, A, Pushpa, K, Khairnar, K, Priya, T, 2010: Polymerase chain reaction confirmation of diagnosis of intestinal amebiasis in Puducherry. Indian J. Gastroenterol. 29:140-2.

Pedraza-Diaz, S, Amar, C, Mc-Lauchlin, J, 2000: The identification and characterization of an unusual genotype of Cryptosporidium from human feces as Cryptosporidium meleagridis. FEMS Microbiol. Lett. 189, 2:189-94.

Petri, WA, Jr, R, Haque, D, Lyerly, Vines, RR, 2000: Estimating the impact of amoebiasis on health. Parasitol. Today 16:320-1.

Shrestha, S, Larsson, S, Serchand, J, Shrestha, S, 1993: Bacterial and cryptosporidial infection as the cause of chronic diarrhea in patients with liver disease in Nepal. Trop. Gastroenterol. 14:55-8.
Snelling, WJ, Xiao, L, Ortega, G, Lowery, C J, Moore, JE, et al, 2007: Cryptosporidiosis in developing countries. J. Infect. Develop. Count. 1: $242-56$

Spano, F, Putignani, L, McLauchlin, J, Casemore, DP, Crisanti, A, 1997: PCR-RFLP analysis of the Cryptosporidium oocyst wall protein (COWP) gene discriminates between $C$. wrairi and $C$. parvum, and between $C$. parvum isolates of human and animal origin. FEMS Microbiol. Lett. 150:209-17.

Stark, D, Al-Qassab, SE, Barratt, JL, Stanley, K, Roberts, T, et al, 2011: Evaluation of multiplex tandem real-time PCR for detection of Cryptosporidium spp., Dientamoeba fragilis, Entamoeba histolytica, and Giardia intestinalis in clinical stool samples. J. Clin. Microbiol. 49, 1: 257-62.

Tuli, L, Singh, DK, Gulati, AK, Sundar, S, Mohapatra, TM, 2010: A multi-attribute utility evaluation of different methods for the detection of enteric protozoa causing diarrhea in AIDS patients. BMC Microbiol. 10:11-6

Vajro, P, Paolella, G, Fasano, A, 2013: Microbiota and gut-liver axis: a mini-review on their influences on obesity and related liver disease. J. Pediatr. Gastroenterol. Nutr. 56, 5:461-8.

WHO 1997: Amoebiasis. Wkly. Epidemiol. Record.72:97-100.

WHO, 2010: Agents Classified by the IARC Monographs, Volumes 1-100" (PHP).

Younes, TA, Hussein, MM, Kamal, SM, Mohamed, DM, 1996: Parasitological and bacteriological studies in recurrent diarrhea in patients with chronic liver disease. J. Egypt. Soc. Parasitol. 26, 3:697-708.

Youssef, FG, Adib, I, Riddle, MS, Schlett, C D, 2008: A review of Cryptosporidiosis in $\mathrm{J}$. Egypt. Soc. Parasitol. 38:9-28

Zebardast, N, Yeganeh, F, Gharavi, MJ, Abadi, A, Seyyed Tabaei, SJ, et al, 2016: Simultaneous detection and differentiation of Entamoeba histolytica, E. dispar, E. moshkovskii, Giardia lamblia and Cryptosporidium spp. in human fecal samples using multiplex PCR and qPCRMCA. Acta Trop. 162:233-8.

Zhang, Z, Zou, S, Fu, L, Cai, L, Jin, L, Liu, Y, et al, 2008: Severe dendritic cell perturbation is actively involved in the pathogenesis of acuteon-chronic hepatitis B liver failure. J. Hepatol. 49: 396-406 